\title{
DVPW-WISSENSCHAFTSPREIS
}

\section{Laudatio: Verleihung des DVPW-Förderpreises für Dissertationen 2013 an Sophia Schubert}

\author{
André Brodocz / Anna Geis / Florian Grotz
}

Der Förderpreis der DVPW für die beste Dissertation 2013 geht an Sophia Schubert, die derzeit als Wissenschaftliche Mitarbeiterin an der Freien Universität Berlin tätig ist. Ausgezeichnet wird ihre Arbeit zum Thema „Die globale Konfiguration politischer Kulturen. Eine theoretische und empirische Analyse“, die 2012 an der Universität Stuttgart auf Grundlage der Gutachten von Prof. Dr. Dieter Fuchs und Prof. Dr. Viktoria Kaina als Promotionsschrift angenommen und noch im selben Jahr bei Springer VS veröffentlicht wurde.

Die Anzahl der eingereichten Arbeiten war mit 14 etwas geringer als in den vergangenen Jahren. Dem üblichen Auswahlverfahren folgend, wurden zunächst Fachgutachten von einschlägig ausgewiesenen Kolleginnen und Kollegen eingeholt, die die Dissertationen nach vorgegebenen Kriterien beurteilen sollten. Auf Basis der Gutachtervoten kam die Jury zu der einhelligen Einschätzung, dass die Arbeit von Sophia Schubert im Vergleich zu ihren Mitbewerber_innen die Vergabekriterien in herausragender Weise erfüllt, wie im Folgenden deutlich wird.

Die Studie verfolgt ein sehr ambitioniertes Ziel: Sie will „die ,Diversitätsforschung' als neuen Forschungsstrang innerhalb der empirisch orientierten politischen Kulturforschung [...] etablieren“ (S. 13), indem sie theoretische Perspektiven der Kultursoziologie und der normativen Politischen Theorie mit dem Mainstream der empirischen Politischen Kulturforschung verknüpft. Diese heterogenen Untersuchungsansätze eint eine grundlegende Frage: Welche Konsequenzen haben kulturelle Gemeinsamkeiten und Unterschiede, sowohl weltweit als auch innerhalb von Staaten, für die Funktionsweise und Legitimität politischer Systeme? Dadurch dass die Autorin die unterschiedlichen Positionen der normativen Diversitätsforschung für die empirische Analyse fruchtbar macht, gelingt ihr nicht nur eine differenzierte Antwort auf die Frage, sondern auch eine theoretisch fundierte Synthese der unterschiedlichen Diskussionsstränge.

Konzeptionell verortet sich die Studie innerhalb des „klassischen“ Paradigmas der Almond/Verba-Schule, indem sie den Begriff „Politische Kultur“ auf seine kognitiven und normativen Dimensionen eingrenzt und diesen auf die Objekte „politische Gemeinschaft“, „polity“ (Regime) und „politics“ (politische Prozesse und Akteure) bezieht. Den einschlägigen Literaturbestand unterteilt die Autorin sehr überzeugend in drei Forschungsstränge, die sich mit der „Persistenz“ politischer Systeme, der „Qualität“ von Demokratien sowie der „Diversität“ politischer Kulturen im internationalen Kontext befassen. Den letztgenannten Zweig sucht sie 
nun eigenständig zu begründen. Dazu nimmt sie die Debatten um einen „Kampf der Kulturen " (Samuel Huntington), ihre weitere internationale Homogenisierung (Ronald Inglehart) oder Hybridisierung (Peter Katzenstein u.a.) als Ausgangspunkt, um eine theoretisch angeleitete und empirisch fundierte „Kartografie“ der politisch-kulturellen Welt zu erstellen.

In theoretischer Hinsicht erscheint die Studie vor allem deswegen als innovativ, weil sie die politiktheoretischen Debatten um „moralische Universalien“, „kulturelle Kontexte“ und „Transkulturalität“ systematisch aufgreift und sie für die empirische Analyse fruchtbar macht. Besonders gelungen ist dabei, wie sie die jüngsten Diskussionen zur „comparative political theory“ (Fred Dallmayr) in die Politische Kulturforschung einbindet.

Empirisch basiert die Studie auf Daten der fünften Welle der „World Values Surveys“ (WVS), die mithilfe quantitativ-statistischer Verfahren untersucht werden. Die empirische Analyse besticht zum einen dadurch, dass die Autorin die Datenlücken akribisch dokumentiert und die daraus resultierenden Einschränkungen und Probleme hinsichtlich der Aussagekraft reflektiert. Zum anderen nutzt sie gekonnt ein neueres methodisches Verfahren, das der „multidimensionalen Skalierung “ (MDS), um ihre empirischen Befunde summarisch zu visualisieren und eine politisch-kulturelle „Weltkarte“ zu erstellen.

Das Ergebnis der Arbeit im Hinblick auf die übergeordnete Fragestellung ist pointiert und differenziert zugleich. Sophia Schubert zeigt, dass die globale Konfiguration politischer Diversitätskulturen keiner der drei Grundpositionen (Einheit, Vielfalt, Hybridität) vollständig entspricht. Auf den einzelnen Analyseebenen werden indes deutliche Muster erkennbar: Während die „universalistische“ Auffassung in der Polity-Dimension dominiert, zeigt sich in Bezug auf die PoliticsDimension eine Vielfalt unterschiedlicher „Kulturkreise“; hinsichtlich der politischen Gemeinschaft schließlich scheint sich eine „hybridisierte“ Weltkultur herauszubilden. Insgesamt liegt damit - in den Worten des Fachgutachters - „ein sehr plausibel dargestelltes, gut dokumentiertes und in jedem Schritt nachvollziehbares Ergebnis [vor], das angesichts der Hitze mancher vorangegangener Debatten in diesem Bereich eine wohltuende Ernüchterung bedeutet“.

Sophia Schubert hat eine Dissertation verfasst, die durchweg ein sehr gut informiertes, systematisches und eigenständiges Vorgehen aufweist. Die Autorin vertritt ihre theoretisch und empirisch fundierte Position überzeugend und in großer Klarheit. Untersuchungsansatz wie Ergebnisse der Studie enthalten zahlreiche Impulse und Anregungen für verschiedene Teilbereiche der gegenwärtigen Politikwissenschaft. Nach Auffassung der Jury hat Sophia Schubert das hochgesteckte Ziel erreicht, einen sowohl theoretisch als auch empirisch bedeutsamen Beitrag zur Erfassung der „globalen Konfiguration politischer Kulturen“ zu leisten. Wir gratulieren der Preisträgerin herzlich und wünschen ihr weiterhin alles Gute und viel Erfolg. Last, but not least ein großer Dank an die Gutachterinnen und Gutachter, die unsere Auswahl mit ihren ebenso sachkundigen wie pointierten Stellungnahmen erheblich erleichtert haben. 